\title{
Cancellation of Space-Based Interference in Radio Telescopes ${ }^{1}$
}

\author{
Lou Nigra $^{2}$ \\ Department of Astronomy \\ University of Wisconsin \\ Madison, Wisconsin
}

\begin{abstract}
A concept is presented that was developed at the National Astronomy and Ionosphere Center (NAIC) at Arecibo, Puerto Rico, for suppression of Global Positioning System (GPS) signals in the $305 \mathrm{~m}$ dish radio receiver path prior to back-end processing. The subsystem requires no auxiliary antenna and is intended for easy integration with virtually any radio telescope system. The design focuses on the GPS L3 signal at 1381.05 MHz which, during periodic tests, interferes with observations of objects in an important range of redshifts. The signal dynamically change modulation modes and our scheme has demonstrated, through simulations using actual telescope data, the ability to acquire and track the signal as well as detect mode changes in order to to apply cancellation or blanking, as appropriate. A follow-up is planned to develop a prototype to deploy and evaluate at NAIC.
\end{abstract}

\section{Introduction}

Navigation satellite systems, such as Global Positioning System (GPS) and the Russian Globalinaya Navigatsionnaya Sputnikovaya Sistema (GLONASS), are inherently troublesome to radio astronomy. Ground-based Radio Frequency Interference (RFI) typically enters the system from near the horizon through antenna side lobes far from the antenna pointing direction where the system response is highly suppressed. Satellite signals can arrive at any angle through closein side lobes with much higher gain. In this work, we describe a concept for mitigating the troublesome GPS signal at L3, which frequently interferes with the operation of neutral Hydrogen (HI) surveys at the National Astronomy and Ionosphere Center's Arecibo observatory (AO), in Puerto Rico.

\section{The Problem}

The problem presented by the GPS L3 signal to radio telescope systems is illustrated in Figure 1, where several GPS satellites are generally close enough to the antenna pointing direction at a given time to produce a strong interfering system response. Sometimes, in addition to the standard L1 signal, the L3 signal is active and while tuned to the range of frequencies interesting

1 NAIC provided support through a pre-doctoral appointment. Partial support was provided through the National Space Grant College and Fellowship Program and the Wisconsin Space Grant Consortium. Partial support was also provided through NSF grant AST 0908134.

2 The following collaborators are acknowledged: B. Murray. Lewis (NAIC), Clyde Edgar (The Aerospace Corporation) Phil Perillat (NAIC), Luis Quintero (NAIC), Snežana Stanimirović (University of Wisconsin Madison) and J. S. Gallagher, III (University of Wisconsin - Madison). 
to HI surveys such as the Arecibo Legacy Fast L-band Feed Array survey (ALFALFA), its interfering signal spectrum overlaps red-shifted $21 \mathrm{~cm} \mathrm{HI}$ emission (1420.4 MHz at rest) from the Coma galaxy supercluster at $\mathrm{z}=0.03$. Making matters worse, its $220 \mathrm{~km} \mathrm{sec}{ }^{2}$ wide spectrum is similar to that of spiral galaxy signatures.

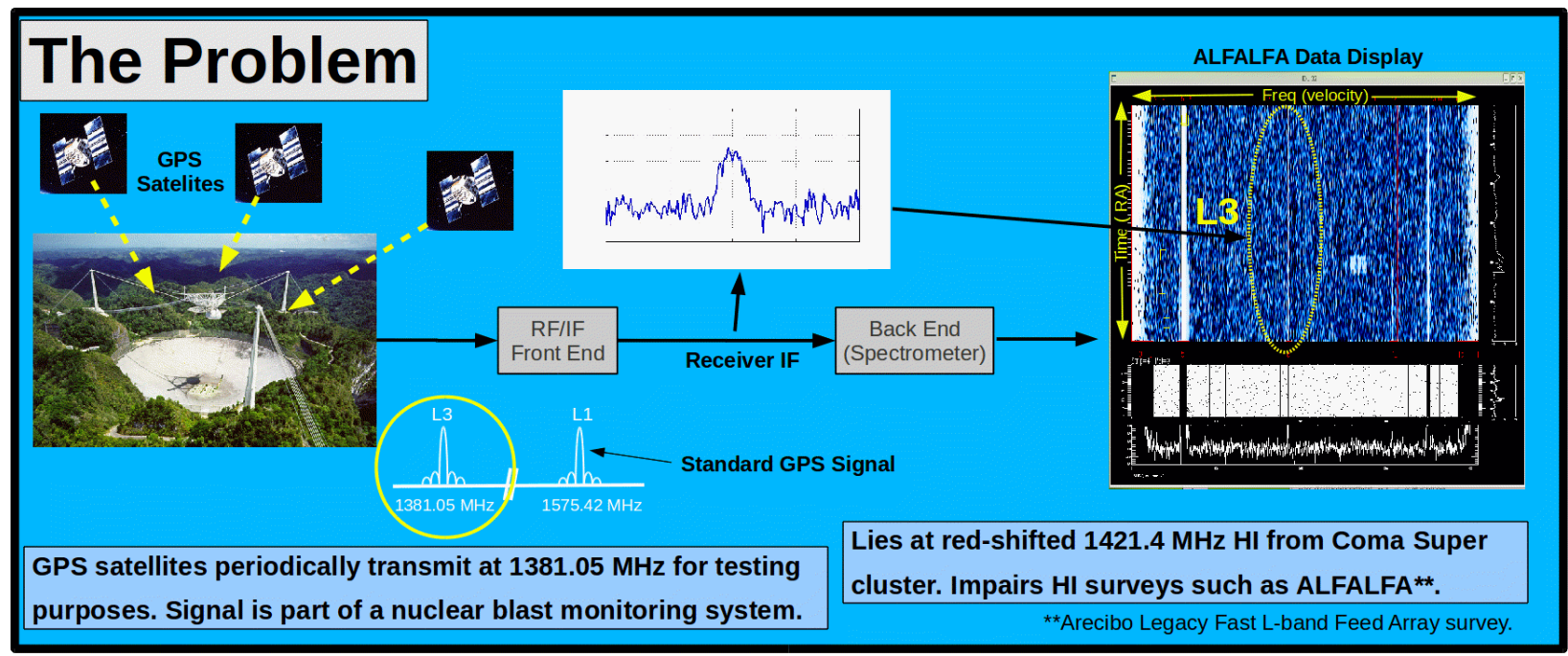

Figure 1 - The GPS L3 Problem.

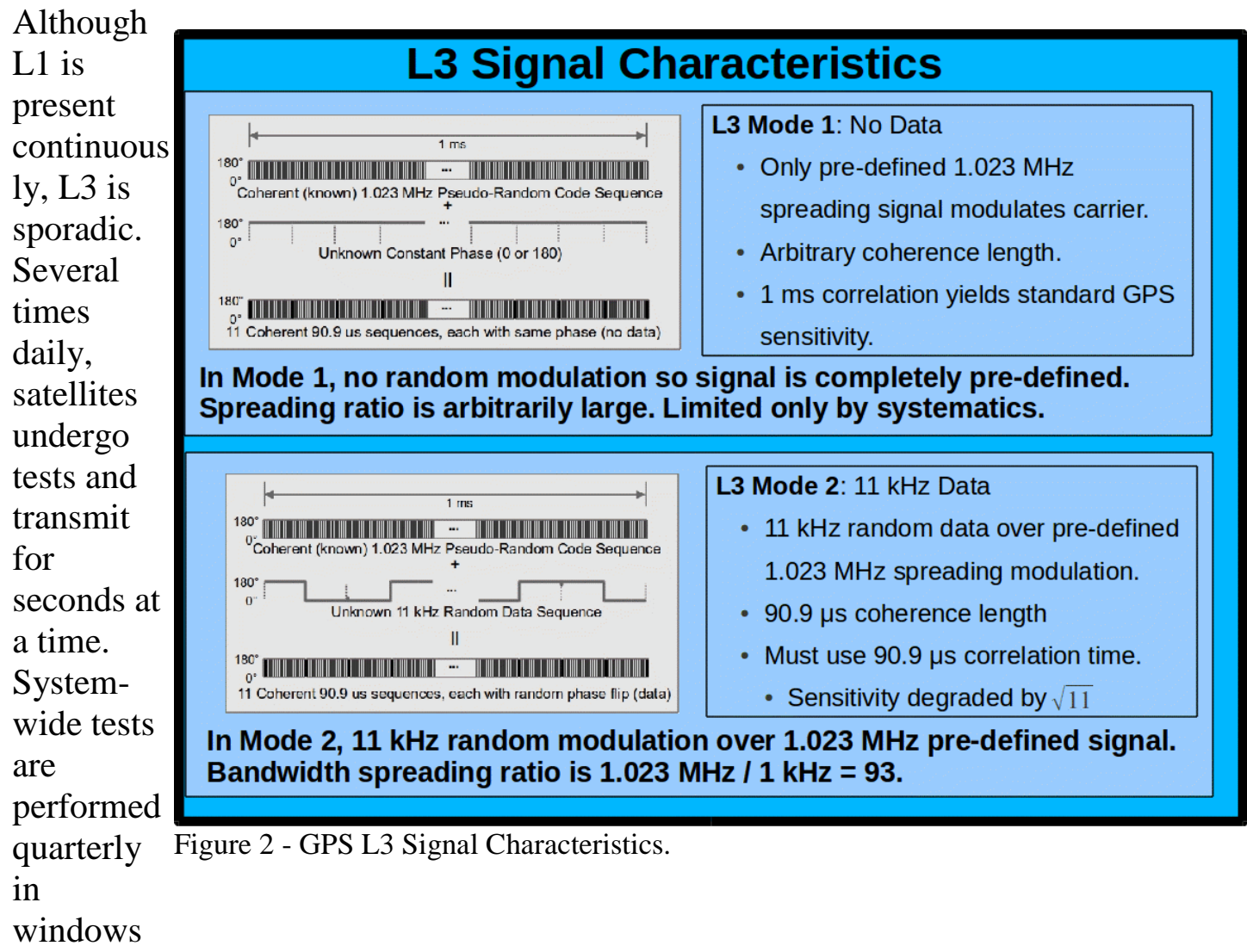


lasting for days where satellites can transmit for minutes at a time. The occurrence and length of transmissions are unpredictable. The authorities coordinate with the radio astronomy community, giving advance notice of these test windows. While transmitting, the signal can operate in two modulation modes, described in Figure 2. The signal is generally in Mode 1, un-modulated by data with only PRN modulation present, but will periodically switch to Mode 2 and overlay its unique $11 \mathrm{kbps}$ data for a number of $1.5 \mathrm{sec}$ slots. Currently, the strategy at AO is to manually flag data when daily tests corrupt observations and the band is avoided entirely during announced system test windows resulting in shutting down surveys for days at a time.

\section{Prior Work}

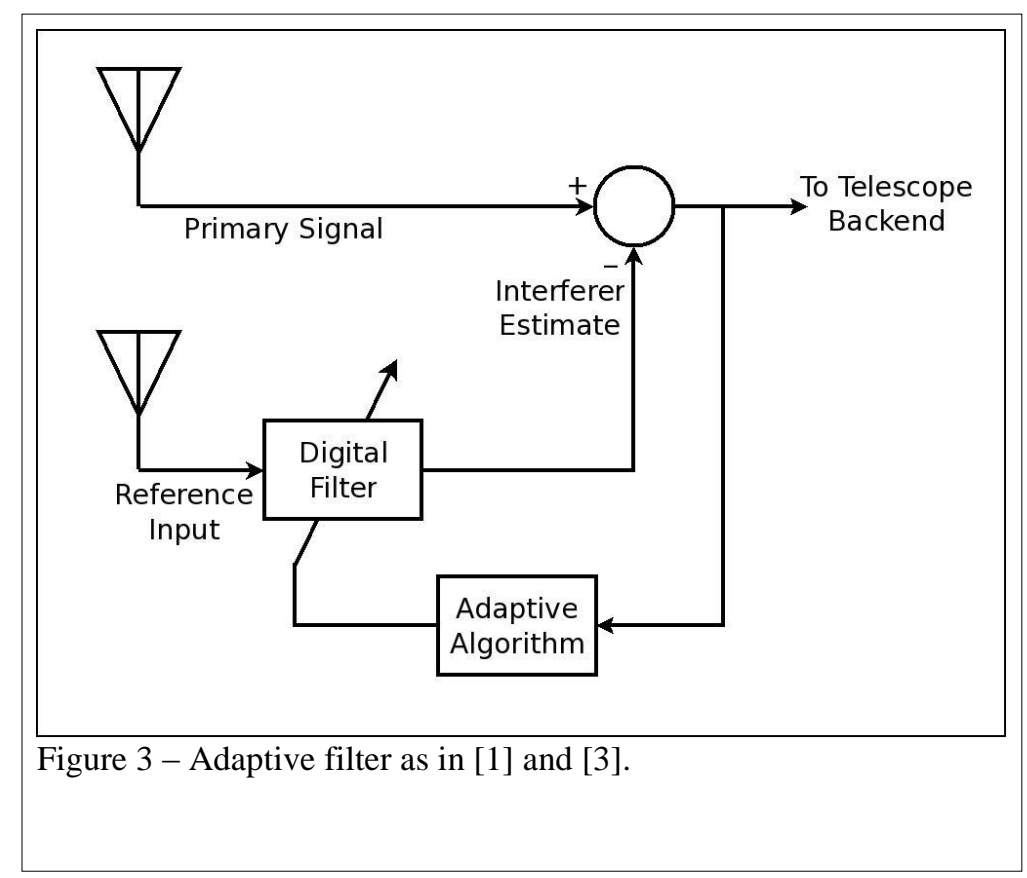

The adaptive filter [4] has a long history in electronic systems and is well-suited for canceling a time-varying, unwanted signal. Figure 3 shows a time domain adaptive filter in a RFI canceling configuration. A high quality replica of the transmitted interferer is required as input and its output is subtracted from the system channel. An algorithm adapts the filter's characteristics to optimally match the channel response by minimizing the interferer after subtraction. In [1], suppression of commercial FM signals with such an adaptive filter was successfully demonstrated. Relevant to the problem at hand, in [3] a similar technique was applied to navigation satellite RFI, successfully suppressing a GLONASS signal. In both of these implementations, a separate antenna is required to obtain a reference signal with much higher signal-to-noise ratio (SNR) than the interference. In [3], a tracking dish is required to track the satellite. Given that 4-11 GPS satellites are in the sky at a given time, it is likely that several satellites produce strong interference at any given time from different directions. In fact, we have detected three GPS satellites in a single 30-second sample signal from the AO telescope. Using the method of [3] would require as many tracking dishes as the number of satellites, making it impractical and is clearly inconsistent with our "turn-key" concept. 


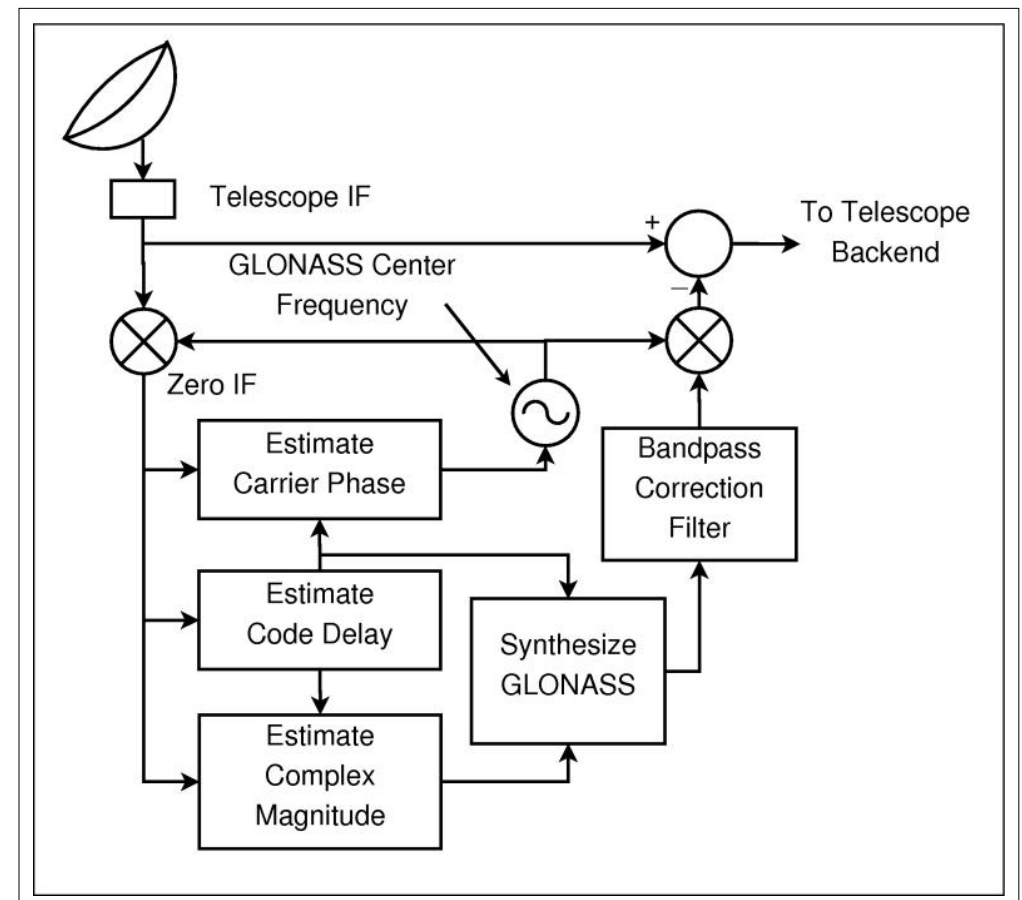

Figure 4 - Active compensation method used in [2].

The need for reference antennas is eliminated for a class of high bandwidth ratio, spread spectrum interferers by a clever approach described in [2], and shown in Figure 4. It exploits the fact that most of the bandwidth of spread-spectrum systems such as GPS and GLONASS is redundant, produced by the pre-defined PRN waveform. In this scheme, the reference signal is obtained from the telescope signal channel itself, the PRN modulation is removed, and the signal is filtered to a lower bandwidth, thus increasing its SNR. This is then noiselessly re-expanded with the PRN, producing the necessary high SNR reference signal. In this case, a frequency domain adaptive filter implementation is used rather than time domain, but the principles are similar. A limitation of thee approach is that with narrower reference bandwidth, group delay in the reference signal filter increasingly de-correlates the replica signal with the interferer, limiting the SNR improvement to about $1 / 10$ of the PRN-to-data bandwidth ratio. In the case of standard GPS (L1), SNR improvement by a factor of 2000 can be obtained, but only by a factor of 9 for L3 with its high data rate. Still, this approach eliminates the multiple antenna requirement and with improvements, serves as the basis for our turn-key subsystem.

\section{The Solution}

The concept is illustrated in Figure 5, which shows RFI Suppression Subsystem (RSS) operation when directly inserted into the existing receiver system at the Intermediate Frequency (IF) stage with no modifications required to the rest of the system. The IF signal is simply diverted to the RSS, which processes the signal, selectively suppressing the undesired GPS signals from multiple satellites while minimally affecting the rest of the signal, and returns the cleaned IF signal to it's original destination, the telescope's Back End Processor. Virtually all radio telescopes have a similar IF stage in their architecture, and the RSS requires minimal automated coordination communication with the telescope control system through standard interfaces, so 
the RSS can be considered a "turnkey" operation, easily integrated with virtually any radio telescope system.

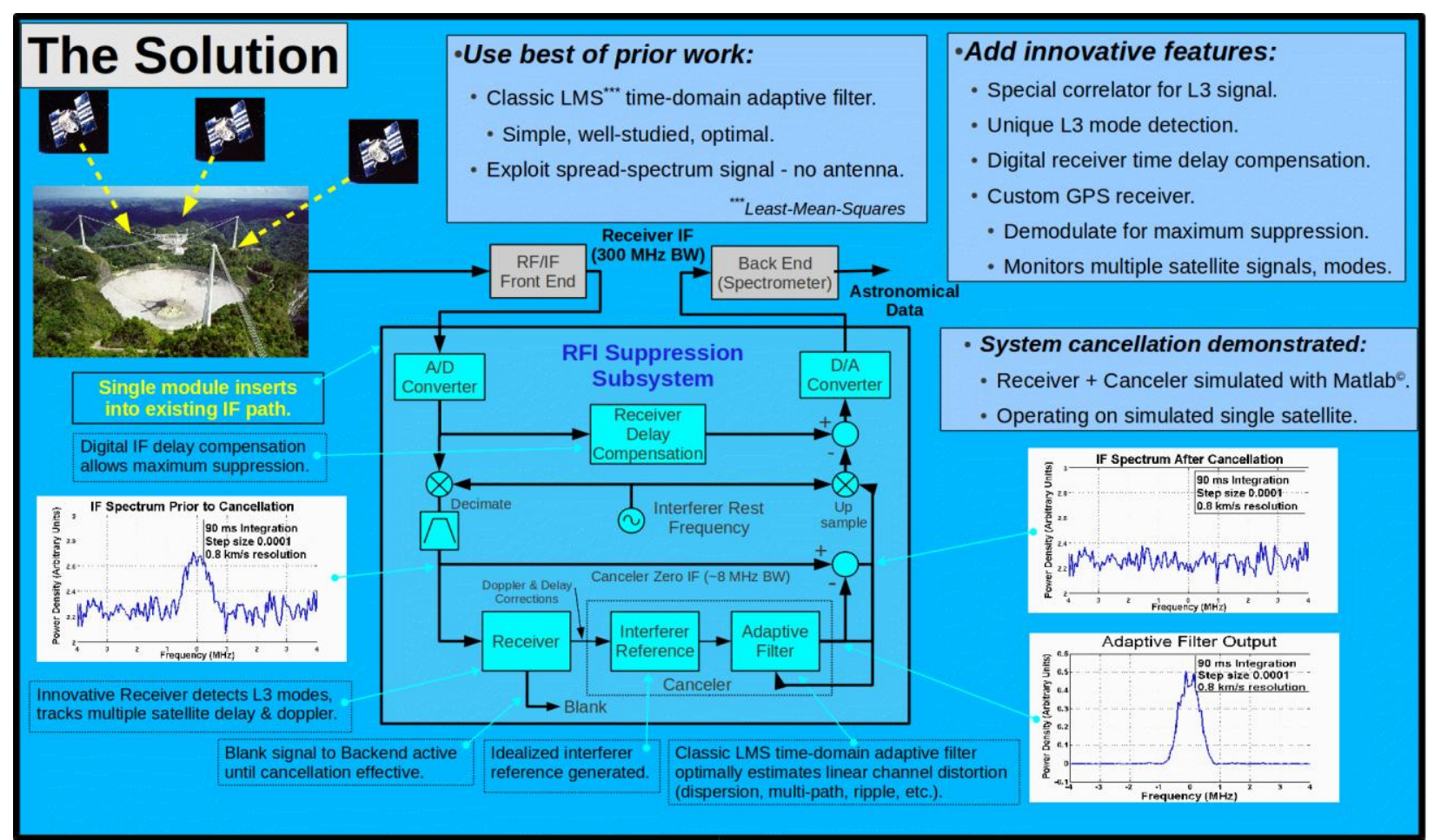

Figure 5 - The GPS Signal Suppression System inserted in the Arecibo radio telescope.

This turnkey capability is in sharp contrast to conventional GPS-type RFI suppression concepts, which require a separate high-gain tracking dish antenna for each satellite to be suppressed. Such a system for one GPS L3 signal is certainly feasible (albeit very expensive), but cannot be considered "turnkey". In fact multiple satellites in different sky positions will produce signals strong enough to interfere with telescope operation, leading to a requirement for several tracking dish antennas, making these approaches completely impractical.

What allows this RSS to avoid the external antenna pitfall is the clever approach devised by [2], mentioned previously, which exploits the spread-spectrum modulation technique used in GPS and other similar satellite systems. Actually, this exploitation is also possible with many other potential ground and space-based interfering signals since direct sequence spread spectrum modulation of some form is commonly used in many radio communications systems, including mobile consumer systems like smart phone networks.

\section{RFI Suppression Subsystem (RSS) Operation}

The subsystem has been successfully simulated with MATLAB ${ }^{\circledR}$. Figure 5 illustrates the detailed operation of the RSS and shows simulator signal spectra produced in the process using realistic simulated inputs. The diverted IF signal from the telescope is digitized with high-speed Analogto-Digital Converters (ADCs). The digitized signal then follows two paths. In one, the signal is 
analyzed and processed to produce a replica of the interfering L3 signal (discussed further below), but to obtain a replica of maximum quality and achieve maximum suppression, the process takes considerable time (10's of milliseconds), so the replica is delayed in time with respect to the incoming interferer. Because of this, in the second path, the digitized IF signal must be delayed by the exact same amount with a simple digital memory-based variable time delay prior to subtracting the replica interferer from it. Compared to previous work $[1,3,2]$ this variable in-line delay feature is unique and allows maximum suppression of the signal by avoiding time delay distortion when subtracting the replica from the signal.

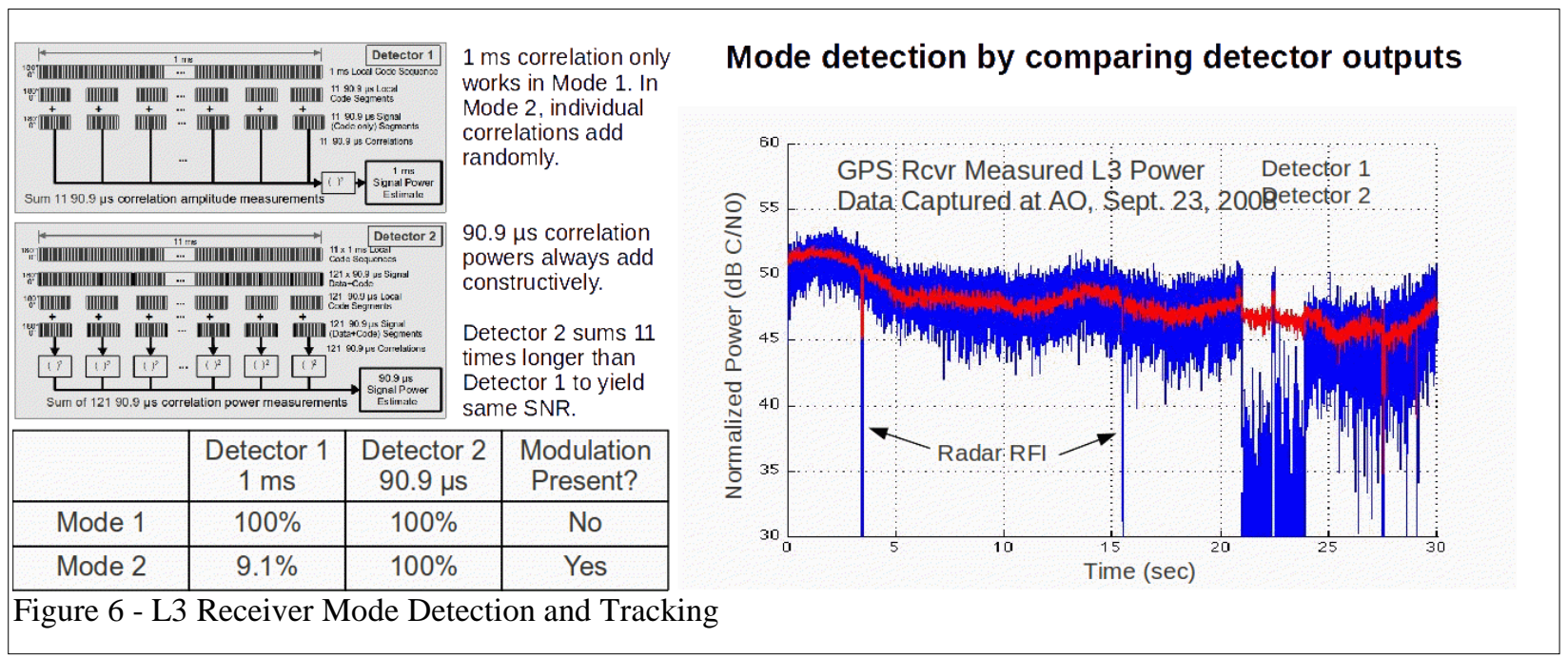

The system produces the interferer replica (graph at lower right) by detection and analysis of the interfering signal (lower left) using an innovative GPS receiver capable of handling the unique L3 modulation characteristics. It produces an idealized interferer reference or replica, which is input to a classical Least Mean Squares (LMS) adaptive filter [4]. The receiver, reference generator and adaptive filter are embedded in a unique frequency down/up conversion (decimation/up-sampling) and signal combining network that allows processing the signal at the desired zero-frequency IF, but translates the interferer-suppressed signal (middle right) back to the exact IF frequency at which it was sampled. The translations account for both the rest frequency and the Doppler shift of the interfering satellite signals. Figure 6 describes the method used to detect both unique L3 modulation modes. This is subsequently used to time-align the receiver with the modulation and also to control suppression strategy. There are two detection algorithms, one of which detects both modes when time-aligned with the signal while the other can only detect Mode 1. This indicates the active mode as well as time alignment, which is used in feedback to track the time delay and Doppler shift of GPS signal. Figure 4 includes a plot of the output of these two signals while the simulated receiver detected and tracked an actual GPS L3 signal throughout a 30-second sample of an actual signal from the Arecibo telescope.

\section{Summary}

The system has demonstrated, through simulations, the ability to detect, identify and track actual GPS L3 signals in both of its modulation modes. It has also demonstrated that it can suppress a single simulated interferer, but the simulator has not been upgraded to include multiple adaptive filter channels, necessary to simultaneously suppress multiple signals. This work is still in 
progress, but is conceptually simple, with high likelihood of success. The programming involved is the main challenge. A second challenge is that for very long (40-second) signal integrations used for sensitive astronomical measurements, there is a small residual structure in the spectrum. This structure, although it would only interfere with the weakest of detectable astronomical signals, is still of concern and must be understood and characterized. It is expected that this effect is precisely predictable and can therefore be eliminated. Work will continue along these lines for the remainder of the year under complementary funding and will culminate in a final peer-reviewed paper and transfer to Arecibo Engineering for further development.

\section{References}

[1] Barnbaum, C., \& Bradley, R. F. 1998, A New Approach to Interference Excision in Radio Astronomy: Real-Time Adaptive Cancellation, 1998 AJ 116, 2598

[2] Ellingson, S., Bunton, J. Bell, J., Removal of the GLONASS C/A Signal from OH Spectral Line Observations Using a Parametric Modeling Technique, 2001 ApJS 135, 87E

[3] Poulsen, A. J., Jeffs, B. D., Warnick, K. F., Fisher, J. R., Programmable Real-Time Cancellation of GLONASS Interference with the Green Bank Telescope, 2005 AJ 130, 2916P

[4] Widrow, B., et al., Adaptive noise canceling: Principles and applications, 1976 Proc. IEEE, 63,1692 